\title{
El concepto de Justicia Global como medio para prevenir la violación de derechos humanos por las industrias extractivas
}

\author{
The concept of Global Justice as a mean to prevent the \\ violation of Human Rights by extractive industries
}

\author{
Patricia Urteaga-Crovetto \\ Pontificia Universidad Católica del Perú \\ purteaga@pucp.edu.pe \\ Yenny Vega Cárdenas \\ Observatorio International de los Derechos de la Naturaleza \\ Presidence.oidn@observatoirenature.org
}

Cómo citar/Citation: Urteaga-Crovetto, Patricia y Yenny Vega. 2021. «El concepto de Justicia Global como medio para prevenir la violación de derechos humanos por las industrias extractivas». Deusto Journal of Human Rights, No. 7: 37-63. doi: http://dx.doi.org/10.18543/djhr.1809.

Sumario: Introducción. 1. Génesis de la teoría de la Justicia Global. 1.1. John Rawls y la genealogía de la justicia global. 1.2. Críticas a Rawls y el surgimiento de la justicia distributiva. 2. La relación entre la justicia global y los problemas locales. 2.1. Los principios para lograr la justicia global. 2.2. Derechos humanos y agentes determinantes para la justicia. 3. Las violaciones contra los derechos humanos y la búsqueda de la justicia global. Conclusión. Bibliografía.

Resumen: La paradoja denominada «maldición de los recursos naturales» ha demostrado que las economías basadas en actividades extractivas no contribuyen positivamente a la erradicación de la pobreza, la discriminación y las desigualdades. Por el contrario, estos problemas parecen empeorar, incrementando los niveles de corrupción, desigualdad y violencia. Mientras que los defensores de estas actividades enfatizan la necesidad de desarrollar las industrias extractivas, sus críticos señalan las consecuencias ambientales, políticas y sociales de esta industria. No obstante, es innegable que la principal causa de los conflictos socioambientales en el mundo, que incluye la violación de los derechos humanos fundamentales, los derechos culturales y los derechos ambientales, tiene como corolario la mercantilización y explotación de la naturaleza. El carácter global de las industrias extractivas requiere una 
teoría de la justicia más comprensiva, que tenga en consideración el desafío global y la soberanía estatal, además de la complejidad que caracteriza a este tipo de problemas en términos de espacio, tiempo, sujetos y materias. Filósofos políticos, en su mayoría angloamericanos, han desarrollado diversas aproximaciones teóricas a la justicia global que hacen referencia al desarrollo, la redistribución, la protección ambiental, la solidaridad, la responsabilidad, la protección de los derechos humanos y la equidad. Este artículo busca analizar en qué medida el concepto de justicia global puede contribuir a comprender mejor y resolver los conflictos socioambientales relativos a las industrias extractivas.

Palabras clave: Recursos naturales, justicia global, industrias extractivas, derechos humanos, equidad, justicia ambiental.

Abstract: The paradox of «the natural resources curse» has shown that extractive economies do not contribute positively to the eradication of poverty, discrimination and inequality. On the contrary, these problems seem to worsen in such economies, increasing the levels of corruption, inequality and violence. While advocates of these activities emphasize the advantages of developing extractive industries, their critics underline the environmental, political and social consequences of this industry. It is undeniable, however, that the main cause of social and environmental conflicts in the world, including the violation of fundamental, cultural and environmental rights, is the commodification and exploitation of nature. The global character of extractive industries demands a more comprehensive theory of justice that considers the global challenge and state sovereignty, as well as the complexity that characterizes this type of problem in terms of space, time, agents and subjects. Political philosophers, mostly Anglo-American, have developed various theoretical approaches to global justice that refer to development, redistribution, environmental protection, solidarity, responsibility, the protection of human rights and equity. This article seeks to analyze the extent to which the concept of global justice can contribute to a better understanding and resolution of socio-environmental conflicts related to extractive industries.

Keywords: Natural resources, global justice, extractives industries, human rights, equity, environmental justice. 


\section{Introducción}

En las últimas décadas, el crecimiento exponencial de las industrias extractivas ha generado preocupaciones respecto a la sostenibilidad ambiental. Desde la década de los noventa, se ha advertido sobre la relación inversa entre recursos naturales abundantes y crecimiento económico, desarrollo y buena gobernanza (Abumere 2015, 104). La paradoja denominada "maldición de los recursos naturales» (Auty 1993, 2; Soros 2007, 2) ha demostrado que las economías basadas en la explotación de los recursos naturales no contribuyen positivamente a la erradicación de la pobreza, la discriminación y las desigualdades. Por el contrario, estos problemas parecen empeorar en contextos de industrias extractivas, incrementando los niveles de corrupción, desigualdad y violencia. Mientras que los defensores enfatizan la necesidad de desarrollar las industrias extractivas, sus críticos señalan las consecuencias ambientales, políticas y sociales de estas actividades.

La devastación ambiental suele estar vinculada a la trasgresión de derechos que no solo afecta la vida, la integridad, la salud y los medios de vida de las personas, sino también sus creencias culturales y prácticas sociales, así como otros derechos humanos fundamentales (Comisión Internacional de Juristas, 2016; Comisión Interamericana de Derechos Humanos, 2020, 2015; Manirabona y Vega, 2019; Svampa, 2019; Sawyer y Gómez, 2012; Atapattu, 2018). Esta problemática se expresa de manera crítica en una tasa relativamente alta de conflictividad que afecta sobremanera a poblaciones reconocidas como vulnerables. En este artículo entendemos por conflicto socioambiental aquella situación en la que dos o más actores (individuos, grupos, estados, empresas, etc.) establecen relaciones de oposición respecto a la naturaleza y los recursos naturales dentro de un rango que incluye desacuerdos y hasta violencia. Estas relaciones, caracterizadas por vulnerabilidades y desigualdades de poder, no sólo comprenden aspectos materiales sobre la contienda sino también simbólicos (Peluzo y Watts 2001; Li 2015; Ide 2016; Martínez-Allier y Walter 2016). Por tanto, los conflictos socioambientales reflejan la transgresión o violación de derechos vinculados a la relación ser humano-naturaleza y las múltiples formas en las que esta relación se expresa. En la medida que trascienden un espacio particular, los conflictos socioambientales, y la violación de derechos humanos concomitante a aquellos, se han convertido en un problema de justicia global.

La reflexión filosófica sobre la justicia global resulta necesaria para enfrentar los problemas que afectan a la humanidad, como 
la comprensión de la dimensión de los problemas ambientales contemporáneos, las fuerzas globales que generan las injusticias respecto a los recursos naturales y las reivindicaciones y discursos sobre las desigualdades (Tan 2014; Martin 2013). Mientras que en la filosofía clásica (Villey 1986) la discusión sobre la justicia se sitúa en el ámbito del Estado, el carácter global de las industrias extractivas demanda una teoría de la justicia más comprensiva que tenga en consideración el desafío global a la soberanía estatal, además de la complejidad que caracteriza a este tipo de problemas en términos de espacio, tiempo, sujetos y materias (Tzevelekos 2014; Hayward e Iwaki 2016). Una teoría contemporánea para «dar a cada persona lo que él o ella merezca» debería trascender la ecuación Estadosociedad, la justicia individual, y aquélla meramente distributiva, de manera que podamos entender cuándo se trata de problemas de justicia global legítimos, cuáles son las responsabilidades por injusticias globales y el alcance de las obligaciones que se deriven de ello (Tzevelekos 2014, 78; Clark, Chhotray y Few 2013; Martin et al. 2013, Tan 2014). En ese sentido, las aproximaciones de la filosofía política y el derecho internacional de los derechos humanos pueden contribuir al establecimiento de la responsabilidad y la rendición de cuentas en aras de la justicia global (Cimadamore 2016).

Filósofos políticos, en su mayoría angloamericanos, han desarrollado diversas aproximaciones teóricas a la justicia global que hacen referencia al desarrollo, la redistribución, la protección ambiental, la solidaridad, la responsabilidad, la protección de los derechos humanos, la igualdad, la democracia y la equidad (Renaut $2013,28)$. Este artículo busca analizar las principales teorías sobre la justicia global que explican los agudos conflictos de gran alcance relativos a las industrias extractivas. La pregunta que guía esta reflexión es: ¿cómo el concepto de justicia global puede contribuir a comprender mejor y resolver los conflictos socioambientales? Para responder a esta pregunta, nuestra investigación se apoya en una metodología de análisis de contenido (L'Écuyer 1990). Así, en la primera parte se desarrolla una parte teórica analítica, que constituye el marco teórico de la investigación. Esta primera parte presenta de manera contrastada un análisis sistemático de los principales aportes de las teorías filosófico-políticas sobre la justicia global. En la segunda parte utilizamos un análisis deductivo analítico, por medio del cual establecemos relaciones entre el caso concreto de las actividades extractivas y la teoría de la justicia global. En la tercera parte procedemos a un análisis cualitativo empírico (L'Écuyer 1990, 106), que nos permite identificar algunos 
casos relacionados con conflictos socioambientales en los que se transgredieron los derechos humanos. Se prosigue con una interpretación obtenida directamente de un análisis cualitativo (L'Écuyer 1990, 109) por medio del cual se evalúa la eficiencia de los remedios jurídicos desde el enfoque de los derechos humanos, y se explora la contribución del concepto teórico de justicia global en la mejor comprensión y resolución de este tipo de conflictos. Las fuentes que usamos provienen de una recopilación, revisión y procesamiento de información documental en la materia. Específicamente, se revisaron algunos casos de jurisprudencia de las cortes regionales de derechos humanos, datos empíricos de fuentes secundarias relacionados a la situación de violación de derechos humanos en distintos países, así como informes realizados por instituciones internacionales de derechos humanos y textos académicos.

\section{Génesis de la teoría de la Justicia Global}

John Rawls es uno de los filósofos contemporáneos más importantes que ha contribuido al debate sobre la justicia global. En su tratado, Rawls (1971) presenta su teoría titulada "Justicia como equidad» en la cual vincula la gobernabilidad con la justicia, afirmando que los conflictos sociales surgen ante la ausencia del sentimiento de este valor (Flores 1999). Rawls presenta dos principios para resolver las injusticias entre ciudadanos de la misma sociedad: el «principio de la libertad», y el «principio de la diferencia» (Rawls 1971, 69). En relación con la justicia internacional, Rawls explica la idea del deber de asistencia que las naciones opulentas tienen en relación con las más oprimidas (Rawls 1971, 106-117).

En respuesta a la teoría de Rawls, los filósofos conocidos como los «igualitarios» recomiendan la igualdad entre nacionales a nivel global y resaltan la necesidad de adoptar mecanismos para redistribuir la riqueza global (Fabre 2005, 139). Los filósofos llamados «cosmopolitas», por su parte, priorizan a los individuos, afirmando que ellos deben disfrutar de sus derechos humanos, sin consideración de su nacionalidad o residencia. Señalan que tener más oportunidades, riqueza o suerte, simplemente porque uno vive en un país más estructurado, es completamente aleatorio. Los resultados terminan siendo injustos. Por ello, debe haber compensaciones para todos los ciudadanos del mundo, de manera que todos puedan tener acceso a oportunidades similares (Pogge 2008, 356). 


\subsection{John Rawls y la genealogía de la justicia global}

John Rawls considera que la justicia es tan importante que su ausencia podría agudizar tensiones y llevar a la guerra. En Justicia como Equidad, el autor menciona dos principios para resolver eventuales injusticias: el principio de la libertad y el principio de la diferencia. El primero alude al disfrute de los mismos derechos y libertades para todos los individuos, mientras que el segundo señala que las diferencias sociales y económicas deben enfrentarse garantizando posiciones laborales a todos los individuos bajo las mismas condiciones, oportunidades y mecanismos de nivelación para los más vulnerables (Rawls 1971, 69).

En relación con el principio de la libertad, Rawls desarrolla el concepto de «posición original» para explicar cómo deberían establecerse los fundamentos de la sociedad bajo el principio de la justicia (Rawls 1971, 69). La «posición original» se refiere al lugar que cada individuo ocuparía una vez que la sociedad se reorganizara. Cuando se realicen decisiones sobre la reestructuración de la sociedad no debería conocerse la posición o el papel de los individuos antes y después de dicho trabajo, que es lo que él denomina el "velo de ignorancia», pues, en la medida que se ofrezcan condiciones para todos sin favoritismo ni prejuicios, se garantizará la igualdad y la objetividad. La asamblea de los representantes debería guiarse solo por el sentimiento de la justicia y el bien común, en la medida que sus miembros no conocerán qué posición ocuparán hasta que la distribución de funciones y beneficios se realice (Rawls 1971, 69).

En El Derecho de Gentes, Rawls (2001, 106-117) explora la idea de la justicia a un nivel internacional recurriendo a sus conceptos sobre la «posición original» y el «velo de ignorancia». Sin embargo, concluye que el "velo de ignorancia» no puede aplicarse a este nivel, porque los miembros de la asamblea de representantes a nivel mundial desempeñan su función de representar a su Estado respectivo. Para Rawls, la justicia internacional es alcanzar la coexistencia pacífica global entre todos los Estados-naciones basándose en la soberanía. A este nivel, la justicia no es un medio para alcanzar la igualdad entre los individuos, sino antes que nada entre las sociedades. Imponer el principio de la igualdad a todos los pueblos correspondería a requerir que todos los países tuvieran una democracia liberal. Por consiguiente, es fundamental la aceptación de las diferencias entre sociedades y tolerar otras formas de gobierno. Si bien para este filósofo, el deber de asistencia permite que las sociedades liberales entren en cooperación con sociedades menos organizadas, ello no implica que la riqueza deba 
redistribuirse, ya que los problemas de las sociedades oprimidas se relacionan con la cultura política. Como consecuencia la redistribución de la riqueza no sería suficiente para rectificar la injusticia política y social (Rawls 2006, 122). Rawls considera que brindar ayuda permite sostener la estructura social de un pueblo oprimido, sin tener que incrementar la riqueza de esa sociedad ilimitadamente, pues la ayuda debe cesar una vez que esta sea capaz de manejar sus propios asuntos de manera racional, $y$, al mismo tiempo, cuente con las posibilidades de crear y preservar instituciones justas. (Rawls 2006, 122). Para Rawls entonces, la redistribución de la riqueza a nivel internacional no es viable, particularmente porque no existe estructura que pueda gestionar tal tarea.

La cultura política en las sociedades oprimidas es crucial para Rawls en la medida que la riqueza de los pueblos depende de sus instituciones, como también de las tradiciones religiosas, filosóficas y morales ligadas a la institucionalidad. Para él, lo que determina la riqueza y el nivel de organización de una sociedad son la ingenuidad y probidad de sus ciudadanos. Las instituciones justas son una precondición para crear prosperidad, lo cual surge de una decisión colectiva para escoger el modo de vida de una sociedad (Rawls 2006, 122). Su tesis sobre la teoría de la «maldición de los recursos naturales» apunta a la ausencia de cultura política, lo que explica por qué los países con menos recursos obtienen plusvalía, mientras que los países con abundancia de recursos naturales tienen grandes dificultades económicas. El autor insiste en que no hay una receta milagrosa; no obstante, la llave para una buena calidad de vida se encuentra en el respeto de los derechos humanos, lo que lleva a mejores gobiernos y sociedades (Rawls 2006, 122). Finalmente, concluye que la paz entre las naciones se logra a través del respeto del principio de la libre autodeterminación de los pueblos, evitando interferir en los asuntos internos de otros Estados.

\subsection{Críticas a Rawls y el surgimiento de la justicia distributiva}

En respuesta a la teoría de Rawls, filósofos en sintonía con un enfoque igualitario (Beitz 2008; Pogge 1994; Pogge y Moellendorf 2008; Barry 2005) proponen la redistribución de la riqueza, así como la reforma de instituciones internacionales. Para estos autores, es imposible imaginar la justicia sin tener en cuenta los desbalances socioeconómicos globales, en los que participan actores globales no gubernamentales. Dada la disminución incremental de la soberanía 
de los Estados-naciones debido a la globalización, la teoría de Rawls se vuelve menos convincente. Para afianzar su riqueza, los Estados industrializados más poderosos interfieren incesantemente en los asuntos de aquéllos más vulnerables para garantizar la dotación de materias primas, mientras los países oprimidos son pauperizados. Esta problemática cuestiona la idea de Rawls sobre la justicia internacional que desestima la necesidad de redistribuir la riqueza.

Los filósofos identificados como «igualitarios» que proponen la igualdad a nivel internacional- critican la postura de Rawls señalando la necesidad de mecanismos para redistribuir la riqueza a nivel global (Beitz 2008; Pogge 1994; Pogge y Moellendorf 2008; Barry 2005). Con base en evidencia de la desigualdad global, consideran intolerable la situación de personas que habitan en una región con ingresos treinta veces mayores que los de los que habitan en otra región, y generalmente, a expensas de los más pobres (Miller 2007, 52). Para eliminar la diferencia abismal entre Estados, estos filósofos proponen eliminar la desigualdad. Thomas Pogge, por ejemplo, plantea crear un mecanismo de redistribución de la riqueza como parte de un modelo que considere que los «Estados cuyos activos per capita están por encima del promedio global pagarían al fondo, y aquellos cuyos activos caigan debajo del promedio se beneficiarían del fondo» (Miller 2007, 58).

Thomas Pogge insiste en el hecho de que la pobreza no está enraizada en la cultura ni en las instituciones, sino que la desigualdad es explicada históricamente. Durante la colonia, la riqueza de los países más ricos se incrementó mediante la dominación y la esclavitud. La acumulación de riqueza por parte de una sola nación y el empobrecimiento de otra se han transmitido de una generación a otra creando un mundo basado en desigualdades. La justicia global no puede ignorar las desigualdades económicas entre sociedades ya que la desigualdad es lo opuesto a la justicia, por lo que un modelo de justicia internacional que no combata las desigualdades existentes no podrá conseguir la justicia entre los Estados-naciones (Pogge 1994, 200). Las diferencias económicas son la base del poder político que influye en la política internacional global, y cuya incidencia debilita dramáticamente a los Estados más vulnerables agudizando su dependencia (Pogge 1994, 196). El principio de tolerancia y neutralidad en las relaciones entre los Estados sugerido por Rawls no puede aceptarse, por lo que Pogge insiste en que el respeto por los derechos humanos sea una regla universal. Una sociedad justa y equitativa no debería mantenerse indiferente ni tolerar las injusticias en un país vecino.

Por su parte, los filósofos conocidos como «Cosmopolitas» conciben la humanidad como un todo (Miller 2007, 25), y por ende 
priorizan a los individuos y la protección de sus derechos fundamentales sin tomar en cuenta su residencia (Pogge 2008, 356). Consideran que el hecho de tener más oportunidades, riquezas y suerte sólo por pertenecer a un país más estructurado es completamente aleatorio e injusto, por lo cual la compensación por cualquier desigualdad debería tener como meta la justicia (Fabre 2005, 145). No podemos ignorar las situaciones que atraviesan las personas que habitan en otras partes del mundo (Miller 2007, 28).

Charles Beitz se pronuncia sobre la justicia en dos niveles: el nivel internacional y el nivel del individuo. En el primer nivel, cuestiona a Rawls por ignorar la soberanía permanente de los Estados sobre los recursos naturales, y sugiere un principio de justicia distributiva internacional absoluta (Beitz 2008, 29). Para aplicar los principios de la justicia distributiva a nivel internacional, el «velo de la ignorancia debe extenderse a todos los asuntos de ciudadanía nacional» (Beitz 2008 , 34). En el segundo nivel, propone lograr la justicia distributiva atribuyendo la posición original a todos los ciudadanos del mundo sin considerar su país de origen. Ningún individuo debería estar orgulloso de tener más talento o más recursos en la medida que su consecución es completamente aleatoria. En suma, mientras los filósofos igualitarios promueven la igualdad entre las naciones y se concentran en la necesidad de mecanismos redistributivos globales (Beitz 2008, 139), los cosmopolitas plantean la justicia individual y la solidaridad a nivel global.

Beitz $(2005,24)$, apoyando la postura de Pogge, reafirma la necesidad de, no sólo mecanismos de redistribución global, sino además de una estructura internacional que garantice tal distribución imparcialmente. De la misma idea es Nagel (2005), quien señala la necesidad de reflexionar sobre la relación entre equidad, justicia socio-económica y soberanía. La justicia global solo puede alcanzarse mediante una soberanía global, puesto que inevitablemente exige la creación de estructuras globales de poder que inicialmente serían ilegítimas, pero que permitirían alcanzar la justicia y equidad universal. Por su parte, Risse (2012) ofrece una compleja estructura teórica que denomina «internacionalismo pluralista» que, entre otros, señala la importancia de vivir bajo cánones de propiedad que permitan satisfacer nuestras necesidades básicas y, especialmente, las de los pobres. Ello será posible si compartimos la idea de una humanidad universal, una estructura institucional común en la que la presencia del Estado no solo es pragmáticamente sino también moralmente conveniente, y, finalmente, un sistema de comercio justo global (Risse 2012; Wettstein 2014). 
Rawls critica las posturas de Pogge y Beitz respecto a la creación de un fondo internacional para la redistribución sin un objetivo o límite máximo. Señala que, dentro de un derecho internacional Westfaliano, este tipo de justicia sólo puede ser fruto de un proyecto consensuado, no un tema contencioso (Heath 2005, 201). Es probable que este convencimiento de Rawls lo haya llevado a excluir a las corporaciones como agentes de justicia global, eximiéndolas así de obligaciones y responsabilidad globales. Como veremos más adelante, las corporaciones generalmente han desarrollado mecanismos voluntarios y autoaplicables que no son obligaciones legales de imperativo cumplimiento frente a las poblaciones afectadas por sus inversiones (Arnold 2013).

Por su parte, Michael Blake lleva el debate más allá mediante el concepto del «deber de la humanidad», por el cual aquéllos que están atravesando dificultades deben recibir ayuda hasta que sus necesidades sean cubiertas. Mediante el concepto del deber de justicia, Blake (2008, 689) apuesta por la institucionalidad proponiendo una estructura (procedimientos, instituciones y normas) que sea justa, equitativa y permanente. Los deberes de humanidad y justicia comprometen a la comunidad global a ayudar a cada país.

Ahora bien, dada la competencia global para la explotación de los recursos naturales que desafía las capacidades regulatorias y el control de los Estados, la soberanía no debería ser un obstáculo para alcanzar la justicia global. A continuación, ofrecemos un análisis de cómo se han desarrollado en los últimos años los principios y las teorías de la justicia global para responder a los conflictos que enfrentan a los Estadosnaciones en la competencia desmedida por la explotación de recursos naturales.

\section{La relación entre la justicia global y los problemas locales}

En el marco de la globalización, los problemas locales se convierten en problemas globales en la medida que su origen y/o consecuencias trascienden las fronteras nacionales (Brincat 2015; Hayward e Iwaki 2016; Martin 2013; Tan 2014). La soberanía estatal ha sido gradualmente erosionada, lo que ha reforzado la interdependencia incremental entre los países (Singer 2002, 2). Por ejemplo, la emigración masiva causada por los conflictos internos y las injusticias demuestran el impacto de las políticas nacionales en la escena internacional. Habermas señalaba las consecuencias de este problema no sólo para las políticas estatales, sino también para lo que conocemos como sociedad nacional (Habermas 2000, 60). 
Los Estados-naciones han perdido autonomía frente a fenómenos como el crimen organizado, las drogas y el tráfico de armas, las evasiones fiscales, el libre comercio, la corrupción y la competencia global por la explotación de recursos naturales. La globalización implica una nueva, irrefutable e irreversible organización internacional. Algunos la vislumbran como parte de la necesidad de repensar un Estado planetario, donde se requiera la colaboración internacional para confrontar desafíos globales ${ }^{1}$.

De todas las causas que impulsan la justicia global en el liberalismo ético, la erradicación de la pobreza extrema debe ser el fundamento que impulse un modelo adecuado de justicia global distributiva (Iglesias 2005). No obstante, existen problemáticas tales como el cambio climático, la violación de los derechos humanos y los impactos ambientales en contextos de extracción de recursos naturales, que también han sido caracterizadas como globales. Lo anterior, nos lleva a considerar la aplicación de nuevas normas y principios, para encontrar soluciones de justicia global que trasciendan los objetivos meramente distributivos, y que tengan como sujeto principal a la humanidad (Brincat 2015; Chaudhuri 2015; Cimadamore 2016; Clark, Chhotray y Few 2013; Martin 2013; Martin et al. 2013; Hayward e Iwaki 2016). En este sentido, los actores o agentes de justicia global como las corporaciones (Arnold 2013), son identificados como causantes activos o pasivos de injusticias que demandan reparación.

\subsection{Los principios para lograr la justicia global}

La reducción de la desigualdad de ingresos global debe ser un requisito para lograr la justicia global. Se trata de un bien común que todas las instituciones, sean nacionales o internacionales, deben proteger y afirmar. Como afirma Nagel, la redistribución de la riqueza es una obligación moral: "la caridad no es suficiente». Es anormal continuar tolerando estas extremas desigualdades que aumentan incesantemente (Nagel 1977, 54-62). Para Buchanan, la

1 Spector (2015) cuestiona la posibilidad de formar una estructura institucional para hacer efectiva la justicia global porque considera que es mero idealismo. Por su parte, Chaudhuri (2015) es escéptico respecto a la posibilidad de formar una estructura internacional de justicia global distributiva puesto que el nacionalismo que permea a los Estado-naciones puede obstruir su formación. En ese sentido, recomienda que la justicia global no se restrinja a la justicia distributiva, sino que se amplíe a temas supranacionales relacionados a la humanidad. En este sentido, véase el capítulo 6 del informe de la CIDH (2020) sobre empresas y derechos humanos. 
estructura básica de la justicia global ya existe, y sus normas han sido reconocidas por los Estados (Buchanan 2002, 698). No obstante, el sistema económico internacional debería reformarse para integrar los principios de equidad internacional (Pogge 2008). Este sistema, creado por países industrializados, incluye instituciones internacionales que eventualmente profundizaron las diferencias económicas entre las naciones ricas y pobres. Si bien el Fondo Monetario Internacional (FMI) y el Banco Mundial fueron fundados para fomentar la ayuda económica, los Estados más poderosos que los controlan han impulsado reformas que favorecen sus propias economías (Pogge 2008, 11-13). La Organización Mundial del Comercio ha contribuido a aumentar las injusticias económicas, dado que su modelo «de libre comercio no es comercio justo» (Pogge 2001, 63). El libre comercio causa asimetría durante las negociaciones de los tratados internacionales, puesto que los países más débiles se sienten presionados a firmar convenios que no necesariamente son en su propio beneficio (Miller 2010, 15). Las empresas multinacionales han sido identificadas como los principales agentes que se benefician de las desigualdades, en las relaciones internacionales (Nagel 1977, 102).

El «enfoque de las capacidades» desarrollado principalmente por Martha Nussbaum (1988, 1992 y 2011) y Amartya Sen (2008) completa la teoría de la Justicia Global, dado que considera la importancia de invertir en la capacidad real de las personas y las sociedades para lograr el bienestar (Monnet 2007). Cinco principios son importantes en este enfoque: tratar a cada persona como un fin, centrarse en la elección y la libertad en lugar de los logros, promover valores pluralistas, demostrar una profunda preocupación por las injusticias sociales arraigadas y atribuir una tarea urgente al Estado de cubrir las necesidades fundamentales insatisfechas como la alimentación, vestido y alojamiento (Nussbaum 2000). Generar capacidades que garanticen una mejor calidad de vida, afirmar la dignidad de toda la humanidad independientemente de las condiciones sociales, culturales o políticas, y respetar los derechos humanos para evitar la perpetuación de la miseria (Nussbaum 2000, 15, 84, 111) son pilares de los derechos humanos.

\subsection{Derechos humanos y agentes determinantes para la justicia}

Una vez categorizado el problema como "global», se identifican los agentes responsables de remediar las injusticias, de acuerdo a cinco parámetros (Stanford Encyclopedia of Philosophy 2015). El «poder»: aquellos que tienen la capacidad de influir y cambiar los 
procedimientos para luchar contra las injusticias, tales como, Estados, jueces y legisladores. En segundo lugar, la «influencia»: aquellos que trabajan en instituciones públicas, puestos de poder o clases sociales, cuyas decisiones pueden influir en el mercado mundial. En tercer lugar, los «intereses»: aquellos que tienen interés en cambiar las estructuras opresivas tienen la responsabilidad de intervenir para remediar las injusticias. La participación de las víctimas en las reformas es fundamental para que estas respondan adecuadamente a sus necesidades. En cuarto lugar, la «capacidad colectiva»: en algunos casos, las organizaciones colectivas tienen la capacidad y los recursos para contribuir a la resolución de conflictos, como asociaciones estudiantiles, organizaciones religiosas, sindicatos, organizaciones no gubernamentales, etc. En quinto lugar, los «beneficios»: a veces, aquellos que crean injusticia o daño difieren de los que se benefician de ellos. Gobiernos que dan la bienvenida a las empresas multinacionales, se benefician de impuestos y flujo de capital, sin participar directamente en violaciones o injusticias. La identificación de los agentes que están moralmente obligados a participar en las soluciones a problemas tan complejos como son las violaciones de los derechos humanos por parte de las industrias extractivas es importante para establecer su nivel de responsabilidad².

\section{Las violaciones contra los derechos humanos y la búsqueda de la justicia global}

Como se mencionó anteriormente, el desarrollo de actividades extractivas suele asociarse con violaciones de derechos humanos y ambientales (Manirabona y Vega 2019). Numerosos casos ilustran esta situación inaceptable que se reproduce en diversos continentes. En el año 2008 dos derrames consecutivos de petróleo, provenientes de las operaciones de la compañía Shell, devastaron las tierras de una comunidad nigeriana de 69.000 habitantes en Bodo, Ogoni. Shell no limpió ni restauró la capa freática ni las tierras agrícolas afectadas. Aún

2 Según David Miller (2001, 453), hay cinco formas de establecer la responsabilidad, o la obligación de ayudar o de intervenir, en la solución de un problema de tan importante índole: la obligación moral de ayudar a una persona necesitada, la obligación resultante de una relación causal, la obligación de intervenir cuando se demuestra que se ha obtenido un beneficio de una situación de vulnerabilidad a pesar de no haber identificado la relación causal, la capacidad de ayudar o de asistir, y la cultura arraigada en la comunidad que crea obligaciones morales de asistencia (Miller 2001, 453). 
más, la compañía ofreció US\$5,000 dólares a la comunidad, 50 sacos de arroz, frijoles, azúcar, tomates y aceite de maní, oferta que el jefe de la comunidad rechazó por considerarla una burla. Como veremos, en febrero 2021 la Corte Suprema de Inglaterra aceptó resolver el caso en su propia jurisdicción (Laville y Akinwotu 2021). En la provincia de Orellana, Ecuador, la empresa ecuatoriana-italiana AGIP Oil aseguró que había llegado a un acuerdo con el pueblo Waorani para perforar pozos de petróleo en sus tierras a cambio de tres quintales de arroz, tres onzas de azúcar, tres sacos de sal, dos pelotas de fútbol, etc. (Saavedra 2011, citado en Urteaga-Crovetto 2018). Las causas de estas situaciones de vulnerabilidad son atribuidas, en parte, a la existencia de gobiernos débiles e incapaces de promover y proteger los derechos humanos (Bannon y Collier 2003, 25), que prefieren cautelar los derechos de las empresas transnacionales dado que estas actividades son sumamente lucrativas (Urteaga-Crovetto 2012, 103-128). Por lo demás, en la mayoría de los casos se ha comprobado que estos ingresos no se distribuyen equitativamente entre la población local, generando conflictos (Bannon y Collier 2003, 4).

En vista de las múltiples violaciones de los derechos humanos relacionadas con las actividades extractivas, se han adoptado diferentes instrumentos de promoción y protección de los derechos humanos entre las empresas, tales como, los Voluntary Principles on Security and Human Rights (2000). Aunque el respeto de este conjunto de principios sea voluntario, Simons y Maclkin (2014) han señalado que el "derecho blando» (soft law) educa a las personas, crea conciencia sobre los impactos y consecuencias de las actividades extractivas, y promueve el respeto de los derechos humanos. Algunas empresas se han comprometido a adoptar estas prácticas; sin embargo, la falta de transparencia sobre su implementación no garantiza que realmente se apliquen los mejores estándares (Simmons y Macklin 2014, 150). Como resultado, la responsabilidad frente a las víctimas de los conflictos se diluye o evade (Reiter 2011, 159).

Diferentes iniciativas han tratado de proponer soluciones frente a este tipo de violaciones: la responsabilidad social corporativa, la adopción de códigos de conducta voluntarios como aquellos sobre Responsabilidad Social Empresarial o Corporativa y los Principios del Ecuador, entre otros. Instituciones que van desde la Organización Internacional del Trabajo hasta el Banco Interamericano de Desarrollo y el Banco Mundial, han adoptado normas para prevenir que las empresas violen los derechos humanos y los derechos ambientales. Algunas guías no vinculantes han sido fundamentales para establecer parámetros para el comportamiento de las multinacionales en lo que 
respecta a las comunidades, como los Voluntary Principles on Security and Human Rights (2000), the UN Global Compact y los Guiding Principles on Business and Human Rights. Estos principios son producto de la labor impulsada por John Ruggie, y fueron adoptados por el Consejo de Derechos Humanos de las Naciones Unidas en junio de 2011. Su objetivo es implementar y poner en práctica el marco de las políticas de las Naciones Unidas que datan de 2008, conocidas como Protect, Respect and Remedy (UN 2008). De conformidad con los principios voluntarios, esta política desarrolla un marco para la protección universal mediante compromisos diplomáticos que tienen como objetivo persuadir, tanto a las empresas como a los Estados, para que respeten normas mínimas de comportamiento. Tres parámetros son esenciales para esta política: los Estados deben proteger los derechos fundamentales individuales y colectivos, las empresas deben tener la responsabilidad social de respetar los derechos humanos, y deben existir mecanismos accesibles para las víctimas cuyos derechos humanos son violados por las empresas o actividades extractivas. Vale la pena señalar que, para las corporaciones, la mayoría de estas normas son hasta la fecha esencialmente voluntarias. Nuestra propuesta se enmarca en la integración tanto a nivel nacional como mundial de una serie de principios vinculantes de justicia global, cuya aplicación y respeto puedan contribuir a resolver tan complejo problema.

Las reflexiones sobre la justicia global distributiva son imperativas para entender las limitaciones que tienen los mecanismos de resolución de conflictos unilaterales, basados exclusivamente en criterios redistributivos o compensatorios ${ }^{3}$. El mecanismo llamado operational level grievance mechanism (mecanismo de quejas de nivel operativo), creado por las empresas extractivas para buscar una solución final a las reclamaciones (Knuckey y Jenkin 2015) se ha implementado en áreas donde se produjeron graves abusos de derechos humanos en concesiones mineras. En la mina de oro Porgera Joint Venture en Papúa Nueva Guinea, Barrick Gold ofreció remediación monetaria a más de 200 mujeres por abusos sexuales ocurridos en el ámbito de la mina, quienes recibieron una compensación financiera a cambio de su renuncia a su derecho a la justicia para demandar a Barrick (Knuckey y Jenkin 2015). Este mecanismo, tal como ha sido usado en algunos casos, socava el derecho a la justicia, la necesidad de establecer la responsabilidad penal de la empresa, y desplaza los esfuerzos

3 Para una crítica de un concepto de justicia reducido al ámbito distributivo en conflictos ambientales, véase Kuehn (2000) y Atapattu (2018). 
estatales para mejorar los procesos de remediación. Habida cuenta de la asimetría incontrastable entre las empresas y las comunidades afectadas, este mecanismo debería someterse a un mayor escrutinio desde un enfoque de derechos humanos, y a estrictas salvaguardias como las propuestas por las Naciones Unidas. Debido a este tipo de casos, la CIDH $(2020,75-6)$ ha señalado lo siguiente:

La CIDH y su REDESCA toman nota de que la existencia de mecanismos alternativos a los judiciales puede facilitar la rendición de cuentas de las empresas y una debida reparación a las víctimas desde etapas tempranas. Sin embargo, si bien en muchos casos pueden actuar como complemento de aquellos mecanismos judiciales, no son asimilables o sustitutos de estos últimos, por lo que la existencia de los primeros no reemplazará la protección judicial que sea requerida según el caso concreto.

En el mismo sentido, el Comité DESC ha manifestado sobre dichos mecanismos extrajudiciales que son un recurso efectivo para las víctimas, siempre que los Estados aseguren que tales mecanismos reúnan al menos las características de garantía indicadas en los Principios Rectores de Naciones Unidas sobre la materia, como son accesibilidad, predictibilidad, transparencia y equidad (CIDH 2020, 75-6). No obstante, la responsabilidad de las empresas que trasgreden derechos humanos sigue siendo esquiva para los sistemas universal y regionales de los Derechos Humanos, lo que redunda en injusticias globales para comunidades locales.

Con base en un análisis cualitativo de los casos sobre empresas multinacionales en la jurisprudencia de la Corte Interamericana de Derechos Humanos, Londoño-Lázaro, et al. (2017) encuentran que las obligaciones que impone la Corte a los Estados provienen principalmente de instrumentos internacionales de soft law (p.ej. United Nations Guiding Principles on Business and Human Rights), mientras otras obligaciones pretenden regular su conducta con buenas prácticas o debida diligencia (consulta ${ }^{4}$, entre otros). Ello puede determinar la ineficiencia de este órgano jurisdiccional debido al carácter no imperativo de algunas responsabilidades que se adjudican a las empresas y a la conducta inescrupulosa de muchas de estas últimas.

Otros autores proponen transformar los códigos de conducta en leyes de imperativo cumplimiento (Cuzacq 2012). Se ha llegado

4 Para una visión crítica sobre el funcionamiento del derecho a la consulta previa, véase Urteaga-Crovetto (2018). 
incluso a proponer la responsabilidad penal de una corporación frente a violaciones de los derechos humanos, compromiso de un Grupo de Trabajo de las Naciones Unidas. Por un lado, este grupo desarrolla guías dirigidas a los gobiernos para exigir e implementar buenas prácticas de las industrias extractivas, que incluyen el enfoque basado en derechos humanos, enfatizando especialmente la participación en la adopción de decisiones ${ }^{5}$, la responsabilidad y la rendición de cuentas (Programa de las Naciones Unidas para el Desarrollo 2018). Por otro lado, desde el enfoque de los Objetivos de Desarrollo Sostenible, se recogen buenas prácticas relacionadas con la prevención y mitigación del deterioro ambiental (Programa de Naciones Unidas para el Desarrollo 2018, ONU y UE, 2010, Woods et al. 2017).

La ONU está evaluando la posibilidad de que las empresas multinacionales sean directamente responsables de las violaciones de los derechos humanos (López 2018). Su objetivo es elaborar una convención internacional vinculante que impida que las empresas seleccionen regiones más complacientes en términos de regulación (Office of the High Commissioner s.f.). Considerando que los derechos humanos tienen un carácter erga omnes, la obligación de protección y de respeto de los derechos fundamentales se extendería a todos los actores involucrados: empresas, Estados, comunidades y también activistas, evitando así la impunidad. En el continente americano se propone la judicialización de las empresas que incurran en violaciones a los derechos humanos y sus aliados transformando principios como la debida diligencia en una obligación legal que prevenga la transgresión de los derechos humanos (Gos 2016; Woods et al. 2017). La Comisión Interamericana de Derechos Humanos (2020), en su informe sobre Empresas y Derechos Humanos, esclarece no sólo los deberes internacionales de los Estados en materia de derechos humanos, sino también las consecuencias que pueden enfrentar las empresas responsables de violaciones.

Recientemente, Canadá ha creado una oficina encargada de recibir denuncias sobre las violaciones de derechos humanos cometidas por empresas extractivas canadienses en otros países, al igual que un órgano consultivo de múltiples partes interesadas para gestionar las

5 Véase la Propuesta de Reglamento del Parlamento Europeo presentada el 14 de octubre de 2020, que modifica el reglamento de la Comisión Europea No. 1367/2006 y del Consejo del 6 de setiembre de 2006, relativo a la aplicación a las instituciones, y a los organismos comunitarios de las disposiciones del Convenio de Aarhus sobre el acceso a la información, la participación del público en la toma de decisiones y el acceso a la justicia en materia de medio ambiente. 
reclamaciones contra las empresas canadienses ${ }^{6}$. Cabe notar que el rol del Ombudsman en responsabilidad social, como lo han denominado, ha sido criticado por la falta de claridad en sus funciones, al igual que por la ausencia de independencia frente al gobierno canadiense, encargado de su nombramiento y remoción (La Presse canadienne 2019) ${ }^{7}$.

A pesar de los esfuerzos desplegados por instancias internacionales como la Organización de las Naciones Unidas y las instituciones del Sistema Interamericano de Derechos Humanos para responder a los impactos de las industrias extractivas, los desafíos parecen sugerir que la solución pasa por profundizar la noción de responsabilidad global de las empresas y los Estados, y evaluar la eficiencia de las estructuras institucionales internacionales. Del mismo modo, los movimientos sociales, así como la academia abogan por la aplicación de normas extraterritoriales que vinculan a las empresas con las leyes de su país de origen en respuesta a sus acciones en comunidades extranjeras (Manirabona 2019; Woods et al. 2017). Esta solución parece haber tenido eco en el caso de Shell en Nigeria, dado que en febrero 2021 la Corte Suprema de Inglaterra aceptó recibir en su propia jurisdicción la demanda relacionada con el caso de derrames de petróleo de Shell (Laville y Akinwotu 2021). Ello refleja, en parte, la crítica jurídica que se desprende de la reflexión de algunos filósofos progresistas sobre la necesidad de una institucionalidad global para lograr la justicia glocal. Una propuesta en ese sentido sugiere que la figura jurídica de la excepción de falta de jurisdicción, para eximir de responsabilidad a los Estados de origen de las empresas transnacionales que transgreden los derechos humanos en un ámbito específico, no debe ser aplicable entre Estados. Aún más, si aquellos Estados pertenecen a un mismo sistema de protección de los derechos humanos, en la medida que comparten un rango de valores dentro de una estructura institucional, ello los hace responsables internacionalmente frente a las prácticas violatorias de sus empresas (Molina-Portilla 2016). Un razonamiento similar yace en la idea de «comunidad política de justicia» basada en la humanidad, que alude a la forma cómo los delitos contra la humanidad

6 Véase Global Affairs Canada (s.f.), Multi-stakeholder Advisory Board on Responsible Business Conduct Abroad. http://www.international.gc.ca/tradeagreements-accords-commerciaux/topics-domaines/other-autre/advisory_bodygroupe_consultatif.aspx?lang=eng. Ver también https://www.international.gc.ca/tradeagreements-accords-commerciaux/topics-domaines/other-autre/faq.aspx?lang=eng

7 Se puede consultar la página web de la Oficina del Ombudsman en el siguiente enlace: https://core-ombuds.canada.ca/core_ombuds-ocre_ombuds/index. aspx?lang=eng. Véase también, Comité québécois femmes et développement (s.f.). 
y el propio Derecho Penal Internacional han contribuido a reforzar esta «comunidad política de justicia» trascendiendo el concepto de soberanía de los Estados (Bernal-Bermúdez 2014). En ese sentido, el juez Kale (2009) propone que, en el caso de crímenes universales, como los delitos de corrupción, la jurisdicción debería ser global. Es decir, cualquier país podría ejercer jurisdicción, capturar y juzgar a los acusados, y de ser hallados culpables, sancionarlos. Finalmente, los estándares del sistema Interamericano de Derechos Humanos sobre la responsabilidad de las corporaciones en sus países de origen por la violación de los derechos humanos en ámbitos locales pueden ser de utilidad con los ajustes ya mencionados (Amaral y Palacio 2018). Es ahora el momento de pasar de la conciencia a la acción con el fin de lograr verdaderamente la justicia global.

\section{Conclusión}

A lo largo de este debate nos hemos preguntado en qué medida el concepto de justicia global puede contribuir a comprender y resolver mejor los conflictos socioambientales. La ecuación que vincula la idea del desarrollo con la práctica de las industrias extractivas ha sido puesta en duda, en la medida que las violaciones de los derechos humanos y ambientales se generalizan y quedan en la impunidad. Las discusiones sobre la justicia global planteadas por la filosofía política contemporánea inciden sobre tres ejes de discusión que pueden iluminar varias dimensiones de los conflictos socioambientales y las violaciones de los derechos humanos: el marco axiológico que fundamenta el concepto de justicia global, el carácter espacial de los conflictos socioambientales, la necesidad de una institucionalidad, normatividad y sanción de carácter global y la responsabilidad por las violaciones de derechos humanos.

Un primer eje que aporta a la comprensión y solución de conflictos socioambientales tiene relación con el tipo de justicia global y el paradigma axiológico que debe sostener este concepto. Por encima de la utilidad pragmática del concepto de justicia global para la problemática socioambiental, destacamos el potencial explicativo del concepto de justicia global sobre las causas de los conflictos socioambientales y la violación de los derechos humanos vinculados a desbalances distributivos a nivel global. Si bien los debates que desarrollan Rawls y sus críticos orbitan sobre la justicia distributiva como aspecto central en la problemática ambiental, las múltiples dimensiones de los conflictos por los recursos y la naturaleza convierten 
en insuficiente esta concepción. Ello ha sido comprobado en algunos conflictos socioambientales que incluyeron violaciones a los derechos humanos, en los que se aplicaron mecanismos voluntarios de las empresas extractivas, centrados exclusivamente en una dimensión material. En ese sentido, un concepto de justicia global debe trascender el paradigma antropocéntrico distributivo, para recoger las preocupaciones interculturales y transgeneracionales sobre el ecosistema. La trascendencia global de estas reivindicaciones, a su vez, evitaría preocupaciones relacionadas con la identidad, el nacionalismo y la dependencia de países pobres que podrían sabotear la vigencia de la justicia global. Preguntarse por valores y principios como la dignidad, la equidad, la redistribución, el desarrollo, la igualdad de oportunidades, la propiedad, la participación, la representación, el reconocimiento, entre otros, tiene sentido sólo si están interrelacionados con la solidaridad transgeneracional, la integridad ecológica y el bienestar de la relación sociedad-naturaleza.

En el segundo eje relativo al carácter espacial de los conflictos, la discusión de la filosofía sobre la justicia global nos remite a tres ámbitos: el del individuo, el del Estado y el internacional. Los conflictos socioambientales entendidos como relaciones sociales respecto a la naturaleza, simbólicas y materiales, opuestas e inequitativas, implican violaciones a los derechos humanos que vinculan los ámbitos antes mencionados. En ese sentido, para la resolución de los conflictos y el establecimiento de las responsabilidades por la transgresión de los derechos humanos, la justicia global debe tener como norte el espacio transnacional.

Finalmente, el tercer eje de discusión asociado a la responsabilidad por las injusticias y la necesidad de una institucionalidad y normatividad global requiere tomar en cuenta la perspectiva multiescalar. En ese sentido, los teóricos de la justicia global señalan la necesidad de establecer estructuras supranacionales que promuevan la democracia y el respeto de los derechos humanos, haciendo imprescindible la participación y la adopción colectiva de decisiones. Los académicos que advierten sobre las deficiencias de los mecanismos existentes en los sistemas de derechos humanos -particularmente, en la determinación y judicialización de las responsabilidades por violaciones a los derechos humanos- consideran imprescindible redefinir las responsabilidades a la luz de un concepto de justicia global y del carácter «glocal» de los conflictos socioambientales que trasciende los límites subnacionales. En la actualidad los organismos internacionales de los derechos humanos vienen explorando diversos mecanismos voluntarios y de soft law para la regulación de las industrias extractivas en espacios institucionales 
internacionales, aunque su incapacidad para hacer efectiva la responsabilidad corporativa y su preocupación centrada en la justicia distributiva suele obliterar otras dimensiones de la justicia ineludibles para las víctimas. Algunas propuestas para prevenir conflictos y establecer la responsabilidad corporativa frente a la trasgresión de derechos humanos, reclaman el fortalecimiento de ámbitos regionales donde los Estados no solo comparten valores comunes, sino también instrumentos normativos internacionales y regionales. La contribución de un concepto integral de justicia global, que trascienda espacios y materias particulares, puede efectivamente contribuir a la comprensión y la prevención los complejos conflictos socioambientales actuales, en la perspectiva de proteger y garantizar la vigencia de los derechos humanos.

\section{Bibliografía}

Abumere, Frank. 2015. Different perspectives on global justice: a fusion of horizons. Bielefeld: Universitätsbibliothek.

Amaral, Amaral do y Viviana Palacio. 2018. «Human rights and extractive industries in Latin America: what responsibility of corporations and their States of origin for human rights violations in the Inter-American Human Rights System?». Revista de Direito Internacional 15, n. 2: 50-71.

Arnold, Denis. 2013. «Global justice and international business». Business Ethics Quarterly 23, n. 1: 125-143.

Atapattu, Saman 2018. "Extractive industries and inequality: intersections of environmental law, human rights, and environmental justice». Arizona State Law Journal 50, n. 2: 431-454.

Auty, Richard. 1993. Sustaining development in mineral economies: The resource curse thesis. Nueva York: Routledge.

Bannon, Ian y Paul Collier. 2003. "Natural resources and conflict: what we can do», en Natural resources and violent conflict, options and actions, dirigido por lan Bannon y Paul Collier, 1-17, Washington: The World Bank.

Barry, Brian. 1995. Why social justice matters. Cambridge: Polity Press.

Beitz, Charles. 2005. "Cosmopolitanism and global justice». The Journal of Ethics 9: 11-27.

Beitz, Charles. 2008. "Justice and international relations» en Global Justice, Seminal Essays, editado por Thomas Pogge y Darrel Moellendorf, 21-48, St. Paul, MN: Paragon House.

Bernal-Bermúdez, Laura. 2014. "Crimes against humanity: global justice and the human rights discourse». Vniversitas 129: 17-38.

Blake, Michael. 2008. «Distributive justice, state coercion, and autonomy» en Global Justice, Seminal Essays, vol. 1, editado por Thomas. Pogge y Darrel. Moellendorf, 657-696, St. Paul, MN: Paragon House. 
Brincat, Shannon. 2015. «Global climate change justice: From Rawls' law of peoples to Honneth's conditions of freedom». Environmental Ethics 37, n. 3: 277-305.

Buchanan, Allen. 2002. "Rawls's laws of people: rules for a vanished Westphalian world», Ethics 110, n. 4: 697-721.

Chaudhuri, Basudeb. 2015. "Identity, nations and the requirements of global justice: limits of the debate in democratic politics». Journal of Interdisciplinary Economics 27, n. 2: 129-151.

Cimadamore, Alberto. 2016. "Global justice, international relations and the sustainable development goals' quest for poverty eradication». Journal of International and Comparative Social Policy 32, n. 2: 131-148.

Clark, Nigel, Vasudha. Chhotray y Roger Few. 2013. "Global justice and disasters». Geographical Journal 179, n. 2: 105-113. https://doi. org/10.1111/geoj.12005

Comisión Interamericana de Derechos Humanos (CIDH). 2015. Pueblos indigenas, comunidades afrodescendientes y recursos naturales: Protección de derechos humanos en el contexto de actividades de extracción, explotación y desarrollo. Washington, DC: Organización de Estados Americanos, Comisión Interamericana de Derechos Humanos.

Comisión Interamericana de Derechos Humanos (CIDH). 2020. Empresas y derechos humanos: Estándares interamericanos. Washington, DC: Relatoría Especial Sobre Derechos Económicos Sociales Culturales y Ambientales. REDESCA. Acceso el 2 de febrero del 2021. http://www.oas. org/es/cidh/informes/pdfs/EmpresasDDHH.pdf

Comisión Internacional de Juristas. 2016. Industrias extractivas, Derechos Humanos y conflictos sociales en Perú Informe de misión. Acceso el 10 de febrero 2021. https://www.icj.org/wp-content/uploads/2016/03/PeruDESC-extractives-Publications-Reports-Facts-Finding-Mission-Report-2016SPA.pdf

Comité québécois femmes et développement (s.f.). L'impact de l'industrie minière et énergétique sur les droits des femmes. AQOCI. Acceso el 7 de febrero 2021. https://aqoci.qc.ca/wp-content/uploads/2015/03/ pdf_impact_de_I_industrie_miniere_sur_les_droits_des_femmes_ particulierement_autochtones_-_cdhal_et_faq.pdf

Cuzacq, Nicolas. 2012. Le cadre normatif de la RSE, entre Soft Law et Hard Law, Acceso el 7 de febrero 2021. https://hal.archives-ouvertes.fr/hal00881860/document

Fabre, Cecile. 2005. «Global distributive justice: An egalitarian perspective». Canadian Journal of Philosophy 31: 139-164.

Flores, Imer. 1999. "El liberalismo igualitario de John Rawls, Cuestiones constitucionales. Revista Mexicana de Derecho Constitucional 1: 86-122.

Global Affairs Canada. 2018. Multi-stakeholder advisory board on responsible business conduct abroad, Acceso el 15 de febrero 2021. http://www. international.gc.ca/trade-agreements-accords-commerciaux/topicsdomaines/other-autre/advisory_body-groupe_consultatif.aspx?lang=eng. 
Gos, Tatiana. 2016. «La 'responsabilidad de respetar' los Derechos Humanos y el establecimiento del deber de debida diligencia como una obligación legal para las industrias extractivas: desafíos y oportunidades en Las Américas», American University International Law Review 32, n. 4: 860893. Acceso el 9 de febrero 2021. http://digitalcommons.wcl.american. edu/auilr/vol32/iss4/6

Habermas, Jürgen. 2000. Après l'État-nation : une nouvelle constellation politique. Francfort-sur-le-Main: Fayard.

Hayward, Tim. y Yukinori. Iwaki. 2016. "Had we but world enough, and time: integrating the dimensions of global justice». Critical Review of International Social and Political Philosophy 19, n. 4: 383-399.

Heath, Joseph. 2005. "Rawls on global distributive justice: a defense». Canadian Journal of Philosophy 31: 193-226.

Ide, Tobias. 2016. "Toward a constructivist understanding of socio-environmental conflicts», Civil Wars 18, n. 1: 69-90, DOI: 10.1080/13698249.2016.1144496

Iglesias, Marisa. 2005. Justicia global y derechos humanos: hacia una ética de las prioridades. Madrid: Universidad Autónoma de Madrid. Acceso el 12 de octubre de 2016. https://www.uam.es/otros/afduam/pdf/9/041_070\%20 marisa\%20iglesias.pdf

Kale, Ndiva Kofele. 2009. «Economic Crimes and International Justice: Elevating Corruption to the Status of a Crime in Positive International Law». Paper presented at the Symposium on Corruption and its Implications for Human Rights. Centre for Human Rights and Democracy in Africa Alliance FrancoCamerounaise Center. Buea, 25 June.

Knuckey, Sarah y Eleanor Jenkin. 2015. "Company-created remedy mechanisms for serious human rights abuses: a promising new frontier for the right to remedy?», The International Journal of Human Rights 19, n. 6: 801-827, DOI: $10.1080 / 13642987.2015 .1048645$

Kuehn, Robert R. 2000. "A taxonomy of environmental justice», The University of Alabama School of Law 30: 10.681-10.793. Acceso el 15 de febrero 2021. https://ir.lib.uwo.ca/cgi/viewcontent.cgi?article=1137\&context=aprci

La Presse Canadienne. 2019. "Ottawa nomme un premier ombudsman de I'entreprise responsable, sans définir ses pouvoirs». Radio Canada. 8 de abril. Acceso el 15 de febrero 2021.https://ici.radio-canada.ca/ nouvelle/1163213/ottawa-nomme-premiere-ombudsman-entrepriseresponsable

Laville, Sandra y Emmanuel Akinwotu. 2021. "Nigerians can bring claims against Shell in UK, supreme court rules». The Guardian. 12 de febrero. Acceso el 15 de febrero 2021. https://www.theguardian.com/ business/2021/feb/12/nigeria-communities-can-bring-claims-against-shelluk-supreme-court-rules

L'Écuyer, René. 1990. Méthodologie de I'analyse développementale de contenu. Québec: Presses de l'Université du Québec.

Li, Fabiana. 2015. Unearthing conflict: corporate mining, activism, and expertise in Peru. Durham: Duke University Press. 
Londoño-Lázaro, Maria Carmelina, Ulf Thoene y Catherine Pereira-Villa. 2017. «The Inter-American court of human rights and multinational enterprises: towards business and human rights in the Americas?», Law \& Practice of International Courts \& Tribunals 16, n. 3: 437-463. https://doi-org. ezproxybib.pucp.edu.pe/10.1163/15718034-12341360.

López, Carlos. 2018. "The road towards a legally-binding instrument in regard to corporations and human rights: from corporate social responsibility to the corporations legal responsibility for human rights violations?», International Commission of Jurists, Acceso el 8 de febrero 2021. http:// opiniojuris.org/2018/07/23/towards-an-international-convention-onbusiness-and-human-rights-part-i/

Manirabona, Amissi. 2019. "Toward barrier removal for transnational human rights litigation in Canadian courts», en Extractive Industries and Human Rights in an Era of Global Justice: New Ways of Resolving and Preventing Conflicts, editado por Amissi Manirabona y Yenny Vega, 39-74, Montreal: Lexis Nexis.

Manirabona, Amissi y Yenny Vega. ed. 2019. Extractive industries and human rights in an era of global justice: new ways of resolving and preventing conflicts, Montreal: Lexis Nexis.

Martin, Adrian 2013. "Global environmental in/justice, in practice: introduction». Geographical Journal 179, n. 2: 98-104.

Martin, Adrian, Shawn McGuire y Sian Sullivan. 2013. "Global environmental justice and biodiversity conservation». Geographical Journal 179, n. 2: 122-131.

Martinez-Allier, Joan. 2002. The environmentalism of the poor. A study of ecological conflicts and valuation, Cheltenham, UK; Northampton, MA: Edward Elgar.

Martinez-Alier, Joan y Mariana Walter. 2016. «Social metabolism and conflicts over extractivism», en Environmental governance in Latin America, editado por Fabio de Castro, Barbara Hogenboom y Michiel Baud, 58-85. Londres: Palgrave Macmillan.

Miller, David. 2001. "Distributing responsibilities», Journal of Political Philosophy 9, n. 4: 453-471.

Miller, David. 2007. National responsibility and global justice. Nueva York: Oxford University Press.

Miller, Richard. 2010. Globalizing justice: The ethics of poverty and power. Nueva York: Oxford University Press.

Molina-Portilla, Diana $M^{a}$. 2016. "Sistema Interamericano, empresas transnacionales mineras y estados de origen: improcedencia de la excepción de falta de jurisdicción entre estados miembros», International Law, Revista Colombiana de Derecho Internacional 29: 57-92. http:// dx.doi.org/10.11144/Javeriana.il 14-29.siet

Monnet, Eric. 2007. "La théorie des 'capabilities' d'Amartya Sen face au problème du relativisme». Tracés, Revue de Sciences humaines 12: 1-14. Acceso el 2 de marzo 2021. https://doi.org/10.4000/traces.211 
Nagel, Thomas. 1977. "Poverty and food: why charity is not enough», en Food policy: The responsibility of the United States in the life and death choices, editado por Peter G. Brown y Henry Shue, 54-62. Nueva York: The Free Press.

Nagel, Thomas. 2005. «The problem of global justice». Philosophy \& Public Affairs 33, n. 2: 113-147.

Nussbaum, Martha. 1988. "Nature, functioning and capability: Aristotle on political distribution». Oxford Studies in Ancient Philosophy 6: 145-184

Nussbaum, Martha. 1992. "Human functioning and social justice: In defense of Aristotelian essentialism», Political Theory 20, n. 2: 202-246.

Nussbaum, Martha. 2000. Women and human development: The capabilities approach, Cambridge: Cambridge University Press.

Nussbaum, Martha. 2011. Creating capabilities. Cambridge, MA: Harvard University Press.

Office of the High Commissioner, Human Rights (s.f.) Working Group on transnational corporations and other business enterprises with respect to human rights. Acceso el 5 de febrero de 2021. https://www.ohchr.org/EN/ HRBodies/HRCMGTransCorp/Pages/IGWGOnTNC.aspx

ONU y UE. 2010. Industrias extractivas y conflicto: Kit de herramientas y orientación para la prevención y gestión de conflictos de tierras y recursos naturales. New York, Equipo Marco Interinstitucional para la Acción Preventiva de la ONU.

Peluso, Nancy L. y Michael Watts. 2001. «Violent environments» en Violent Environments, editado por Nancy L. Peluso y Michael Watts, 3-38, Ithaca: Cornell University Press.

Pogge, Thomas y Darrel Moellendorf. 2008. Global Justice, Seminal Essays, vol. 1. Saint Paul, MN: Paragon House.

Pogge, Thomas. 1994. "An egalitarian law of people». Philosophy and Public Affairs 23, n. 3: 195-224.

Pogge, Thomas 2008. World poverty and human rights: Cosmopolitan responsibilities and reforms, $2^{\circ}$ ed. Cambridge: Polity.

Pogge, Thomas 2001. "Eradicating systemic poverty: Brief for a global resources dividend», Journal of Human Development 2, n. 1: 2-29.

Programa de las Naciones Unidas para el Desarrollo. 2018. Buenas prácticas de extracción: Una Guía para que Gobiernos y asociados puedan integrar el medio ambiente y los derechos humanos en la gobernanza del sector minero. Acceso el 8 de febrero 2021. https://www.undp.org/content/dam/ undp/library/Sustainable\%20Development/Environmental-GovernanceProject/UNDP-MINING\%20Summary\%20Report\%20ES.pdf

Rawls, John. 1971. A Theory of Justice. Cambridge, MA: Harvard University Press.

Rawls, John. 2001. The Law of Peoples. Cambridge, MA: Harvard University Press.

Rawls, John. 2006. Paix et démocratie: Le droit des peuples et la raison publique. Montréal: Boréal. 
Reiter, Sandra 2011. «Moral loopholes in the global economic environment: why well-intentioned organizations act in harmful ways». Éthique et économique/Ethics and Economics 8, n. 1: 154-167. Acceso el 9 de febrero 2021. http://ethique-economique.net/

Renaut, Alain. 2013. Un monde juste est-il possible? Paris: Stock.

Risse, Mathias 2012. On global justice. Princeton: Princeton University Press.

Sawyer, Suzana y Edmund T. Gómez. eds. 2012. The politics of resource extraction. Indigenous peoples, multinational corporations, and the State. Londres: Palgrave Macmillan, UNRISD.

Sen, Amartya. 2008. «Equality of what?» en Global Justice, Seminal Essays, editado por Thomas Pogge y Darrel Moellendorf. 61-82, Saint Paul, MN: Paragon House.

Simmons, Penelope y Audrey Macklin. 2014. The governance gap: Extractive industries, human rights, and the home state advantage. Abingdon: Routledge.

Singer, Peter. 2002. One world: The ethics of globalization. New Haven: Yale University Press.

Soros, George. 2007. "What is the problem with natural resource wealth?», en Escaping the Resource Curse, editado por Macartan Humphreys, Jeffrey D. Sachs y Joseph E. Stiglitz, 1-20. Nueva York: Columbia University Press.

Spector, Horacio. 2015. «Two conceptions of justice and the dystopia of global justice». San Diego Law Review 52, n. 5: 1077-1121.

Stanford Encyclopedia of Philosophy. 2015. "What global duties do we have?». Global Justice, Acceso el 12 de enero 2021. https://plato.stanford. edu/entries/justice-global/

Svampa, Maristella. 2019. Neo-extractivism in Latin America: Socioenvironmental conflicts, the territorial turn, and new political narratives. Cambridge: Cambridge University Press. doi:10.1017/9781108752589

Tan, Kok-Chor. 2014. «Why global justice matters». Journal of Global Ethics 10: 128-134.

Tzevelekos, Vassilis. 2014. «Reconstructing the effective control criterion in extraterrestrial human rights breaches: Direct attribution of wrongfulness, due diligence, and concurrent responsibility». Michigan Journal of International Law 36: 129-178.

United Nations Global Compact (s.f.) El poder de los principios. Acceso el 7 de febrero 2021. http:// www. unglobalcompact.org

United Nations. 2008. Protect, respect and remedy: A framework for business and human rights, Report of the Special Representative of the SecretaryGeneral on the issue of human rights and transnational corporations and other business enterprises, UN Doc.A/HRC/8/5. Acceso el 15 de enero 2021. https://www.business-humanrights.org/sites/default/files/reportsand-materials/Ruggie-protect-respect-remedy-framework.pdf.

Urteaga-Crovetto, Patricia. 2012. "The broker state and the 'inevitability' of progress: Impacts of the Camisea Project on Indigenous People in Peru», en The Politics of Resource Extraction. Indigenous Peoples, Multinational 
Corporations and the State, editado por Suzana Sawyer y Edmund T. Gómez, 103-128. New York. Palgrave-MacMillan.

Urteaga-Crovetto, Patricia. 2019. "Rituals for dispossession: Indigenous Peoples, oil and negotiations in the Peruvian northern Amazon basin». En Extractive industries and human rights in an era of Global Justice. New ways of resolving and preventing conflicts, editado por Manirabona, Amissi y Yenny Vega Cardenas, 267-296, Toronto: LexisNexis.

Urteaga-Crovetto, Patricia. 2018. «Implementation of the right to prior consultation in the Andean countries. A comparative perspective». Journal of Legal Pluralism and Unofficial Law, 50-1: 7-30. Acceso el 5 de febrero de 2021. DOI: 10.1080/07329113.2018.1435616

Villey, Michel. 1986. Philosophie du droit. Paris: Dalloz.

Voluntary Principles on Security and Human Rights (2000). The voluntary principles iniative. Acceso el 7 de marzo 2021. https://www. voluntaryprinciples.org/

Wettstein, Florian. 2014. "On global justice». Business Ethics Quarterly 24, n. 4: 627-630. DOI: 10.5840/beq201424421.

Woods, Cindy, Katharine Valencia y Daniel Cerqueira. 2017. Industrias extractivas y planes nacionales de acción (PNAs) sobre empresas y derechos humanos. Washington DC: Icar. Acceso el 15 de febrero de 2021. http:// www.dplf.org/sites/default/files/final_icar_dplf_extractive_sector_naps_ guidance_espanol_final_reformatted_bn_kv.pdf. 



\section{Copyright}

Deusto Journal of Human Rights / Revista Deusto de Derechos Humanos is an Open Access journal; which means that it is free for full and immediate access, reading, search, download, distribution, and reuse in any medium only for non-commercial purposes and in accordance with any applicable copyright legislation, without prior permission from the copyright holder (University of Deusto) or the author; provided the original work and publication source are properly cited (Issue number, year, pages and DOI if applicable) and any changes to the original are clearly indicated. Any other use of its content in any medium or format, now known or developed in the future, requires prior written permission of the copyright holder.

\section{Derechos de autoría}

Deusto Journal of Human Rights / Revista Deusto de Derechos Humanos es una revista de Acceso Abierto; lo que significa que es de libre acceso en su integridad inmediatamente después de la publicación de cada número. Se permite su lectura, la búsqueda, descarga, distribución y reutilización en cualquier tipo de soporte sólo para fines no comerciales y según lo previsto por la ley; sin la previa autorización de la Editorial (Universidad de Deusto) o la persona autora, siempre que la obra original sea debidamente citada (número, año, páginas y DOI si procede) y cualquier cambio en el original esté claramente indicado. Cualquier otro uso de su contenido en cualquier medio o formato, ahora conocido o desarrollado en el futuro, requiere el permiso previo por escrito de la persona titular de los derechos de autoría. 